\title{
Whither peptide receptor radionuclide therapy for neuroendocrine tumors: an Einsteinian view of the facts and myths
}

\author{
Vikas Prasad • Lisa Bodei • Mark Kidd • Irvin M. Modlin
}

Published online: 8 May 2014

(C) Springer-Verlag Berlin Heidelberg 2014

\section{Introduction}

One should always be cautious of undertaking a rigorous examination of a subject lest hubris interferes with balance and the confidence of "experience" undermines the rigor of facts. Notwithstanding these caveats, this missive seeks to cast a critical light on what, to date, has proven to be a contentious subject: the utility of peptide receptor radionuclide therapy (PRRT) in the management strategy of neuroendocrine tumors (NETs). Mindful of the Einsteinian adage-_"whoever undertakes to set himself up as a judge of Truth and Knowledge is shipwrecked by the laughter of the gods" [1] — we propose to provide a balanced assessment of the matter from the perspective of evaluators of the therapy as opposed to purveyors.

In NET management, a plethora of therapeutic options (some in nascent form, others in "developing phase," and a few with "clinically approved" status) has emerged. The surfeit reflects two issues: firstly the multifaceted challenges posed by NETs (heterogeneous, functional, and protean in their behavior [2]) and secondly the substantial limitations of each of the different therapeutic strategies [3, 4]. This has led to a change in the "end point" of efficacy of a treatment thereby accommodating different visions of efficacy [5]. No longer is the overall survival (OS) of homo sapiens "with

\author{
V. Prasad \\ Department of Nuclear Medicine, Campus Virchow-Klinikum, \\ Charité University Hospital, Berlin, Germany \\ e-mail: vikas.prasad@charite.de \\ L. Bodei \\ Division of Nuclear Medicine, European Institute of Oncology, \\ Milan, Italy \\ L. Bodei $\cdot$ M. Kidd $\cdot$ I. M. Modlin $(\bowtie)$ \\ Department of Gastroenterological Surgery, \\ Yale School of Medicine, New Haven, CT, USA \\ e-mail: imodlin@irvinmodlin.com
}

NET" a principal matter of concern rather how long can a "drug/agent" contest the biological menace of NETs \{euphemistically referred to as progression-free survival (PFS) has taken the centre stage [5]\}. The paradox of increased PFS without increase in OS remains to be explained!

In the eyes of a clinician (or subjected to the scalpel of a surgeon), NETs are generally not dissimilar to other cancers, except in their production of biologically active agents that generate distant and often protean symptoms [2]. These symptoms can be controlled by agents directed towards somatostatin (SST) receptors which are over expressed in 80-85\% of well to moderately differentiated NETs [6-8]. The available agonists against these receptors have an "excellent" effect on the symptom control, by inhibiting the release of bioactive agents and exhibit a relatively good therapeutic index [9]. However, response is not durable and resistance/ tachyphylaxis may evolve over time [10], necessitating dose adjustment or alternative therapies. In addition, the antitumor effects of SST analogs have been reported in a prospective study [11]. These effects are, purportedly due to increased apoptosis, decreased growth and proliferation through mitogen-activated protein kinase, inhibition of insulin-like growth factor receptor signaling, and inhibition of protein synthesis caused by decreased transcription [12-16]. The antitumor effect (measured by an increase in PFS), as shown prospectively in the PROMID study, is, however, only evident in tumors with a proliferation rate $(\mathrm{Ki}-67)<2 \%$ and $<10 \%$ liver involvement (the method of assessment of $10 \%$ liver involvement was at best ambiguous) [11]. More recently, a study (CLARINET) using an alternative SST analog has supported the assertion of proliferative regulation by this class of agents [17].

The other two novel targeted therapies, mTOR inhibitors and tyrosine kinase inhibitors, face a substantial challenge in their general acceptance despite approval for clinical use by the regulatory authorities $[4,18]$. These include substantial 
toxicity rates, a lack of clear understanding of the mechanism of action, questionable (unproven) omnipresence of the targets in NETs, and sometimes tachyphylaxis [19]. As a consequence, physicians have exhibited restraint in using these agents as first-line therapy in advanced pancreatic NETs.

Nonspecific chemotherapeutic regimes including streptozotocin in combination with 5-fluorouracil (FU) or temozolomide in combination with capecitabine have yielded promising results in a "select" subgroup of pancreatic NETs $[20,21]$. For grade 3 neuroendocrine cancers, even fewer treatment options are available, and conventional archaic cytotoxic chemotherapy schemes are usually applied [5, 22].

PRRT, a targeted therapy, has been available for almost 20 years. If pretreatment assessment establishes the presence of adequate SST receptors on tumors, either by use of SST receptor scintigraphy or ${ }^{68} \mathrm{Ga}$ SST receptor positron emission tomography $(\mathrm{PET}) / \mathrm{CT}$, treatment can be considered feasible [23]. Under such circumstances, radionuclide-labeled SST analogs, e.g., ${ }^{177}$ Lu-DOTATATE, can be used to deliver cytotoxic radiation doses to the tumor with consistent cumulative absorbed doses, reaching, in several cases, values up to 250 $300 \mathrm{~Gy}$. The absorbed doses required to eradicate a human cell range widely depending on a series of variables. These include the phase of the cell cycle (dividing cells being the most radiosensitive) but also the intrinsic radiosensitivity of the cell type, the proliferation kinetic, the proportion of clonogenic stem cells, and the DNA repair capacity [24]. For NETs undergoing PRRT, it has been calculated that an absorbed dose of at least 70-80 Gy is required to obtain an objective response [25]. Thus, the combination of a docking mechanism (SST receptors) and a lethal radiation dose theoretically enables effective selective killing of well to moderately differentiated NETs.

\section{PRRT: the facts}

\section{Effectiveness}

"Only the one who does not question is safe from making a mistake" (A. Einstein, letter to Gustav Bucky, 1945).

\section{Efficacy}

Amongst the cytotoxic systemic therapeutic options currently available, PRRT appears to outperform others in terms of efficacy measurable in terms of symptom control to prolongation of life. With ${ }^{177}$ Lu-DOTATATE, patients are expected to live for a median duration of 46 months after first treatment, and disease progression from the time of first PRRT can be delayed for a median duration of 33 months. An even more relevant parameter for efficacy of any treatment, i.e., OS, indicates a survival benefit of 40-72 months from the time of first diagnosis compared to historical controls (suboptimal, albeit only available reference point) [26]. Patients are expected to live for 96.4 months after the first diagnosis if treated with ${ }^{90}$ Y-DOTATOC [27]. Additionally, patients responding to PRRT (morphological, clinical, or biochemical) and qualified for re-treatment are expected to have a benefit of 20 months in OS after first diagnosis; using the time of first treatment as the reference point, patients showing a response were likely to live for approximately 2.8 times longer than the nonresponders [27].

\section{Safety}

"A ship is always safe at the shore - but that is NOT what it is built for" (A. Einstein).

As is true for any cytotoxic therapy, PRRT is not without adverse events. Most are self-limiting and reversible, and are either subacute or acute, such as nausea (or more rarely vomiting), fatigue, mild hair loss (with Lu peptides), impairment of male fertility, and, occasionally, exacerbation of clinical syndromes [31]. The long-term chronic and permanent effect of radiation on kidney and bone marrow is, however, a major concern especially because NET patients have a relatively high OS.

\section{Nephrotoxicity}

The high incidence of renal toxicity, which is especially prominent for ${ }^{90} \mathrm{Y}$ peptides (3-9 \% G4 toxicity [27, 32], is less of a clinical problem after ${ }^{177} \mathrm{Lu}$-octreotate $(0.4 \%$ incidence of G4 toxicity) [26]. Several postulates have been proposed to explain PRRT nephrotoxicity. ${ }^{90} \mathrm{Y}$, based on its beta emission profile, has a longer path length compared to ${ }^{177} \mathrm{Lu}$. In the kidney, radiopeptides are filtered, absorbed in the proximal tubular cells, and transported into the interstitium. Beta particles from ${ }^{90} \mathrm{Y}$ have a maximum path length of $11 \mathrm{~mm}$ and an effective path length of $5.3 \mathrm{~mm}$, thus $90 \%$ of its energy is absorbed within a sphere with a 5.3-mm radius [33]. This path length corresponds to 100-200 cell diameters, making it more probable for them to reach the glomerulus from the proximal tubule. The possibility of damage to the glomerulus with ${ }^{90} \mathrm{Y}$-labeled radiopeptides is thus higher. The risk of radiation-induced nephrotoxicity is to a minor extent predictable based on preexisting factors including age ( $>70$ years), uncontrolled diabetes mellitus and hypertension, and a decreased renal function prior to treatment (e.g., prior treatment with chemotherapies) [34]. Studies correlating dosimetry with clinical results indicate without these risk factors a cumulative 
kidney absorbed dose of up to 40 Gy can be administered. In the presence of risk factors, reduction to $28 \mathrm{~Gy}$ is considered prudent and reasonable [35]. These data have shown that information derived from external radiation therapy cannot be applied to PRRT, given the different rate of dose delivery and the different mechanism(s) of renal irradiation. An individualized PRRT approach based upon dosimetry has been suggested as the optimal strategy if 23 Gy is maintained as the upper renal limit since this cumulative dose may be achieved in two therapy cycles in some while in others the therapy cycle can be extended up to ten cycles [36].

\section{Hematotoxicity}

The most common subacute side effect is transient and mild hematological toxicity, usually occurring with a nadir of 4 6 weeks after therapy. Severe, grade 3 or 4 hematological toxicity occurs in less than $15 \%$ [26, 27, 37, 38]. A highgrade potentially life-threatening bone marrow toxicity (myelodysplasia) is reported in up to $2 \%$ [39-41]. An association with prior chemotherapy or radiotherapy has been implicated and may represent a confounding factor in assigning a causal relationship [31]. A sufficient time interval between chemotherapy and PRRT is currently recommended to avoid such complications. However, mathematical modeling of clinical factors predictive of toxicity indicates that such analysis is less than $50 \%$ accurate [42]. It seems likely therefore that genomic analysis will be required to identify and predict specific organ sensitivity to irradiation [42].

\section{PRRT: myths}

"Once we accept our limits, we go beyond them" (A. Einstein).

\section{Mechanism of action of radionuclides}

According to the principles of radiobiology, free radicals produced during interaction of radiation in and around cells are effective in inducing double-stranded DNA break and consequent apoptosis by targeting G2/M cells. At this time point in the cell cycle, the DNA repair mechanism is least effective. In indolent and slowly progressive tumors, like NETs, a minority of tumor cells are in this phase. A prolongation of the targeted absorbed dose (over several days) has, therefore, a higher probability of reaching radiosensitive cells.

Nuclear medicine therapy owes its success and existence to the presence of a sodium iodide symporter (NIS) on thyroid cells and to the availability of isotopes of iodine, like ${ }^{131} \mathrm{I}$, synthesized in 1938 [43]. In metastasized differentiated thyroid cancers, two thirds of which exhibit radioiodine uptake following thyroid-stimulating hormone (TSH) stimulation, radioiodine therapy can achieve a complete remission in at least one third [44]. Radioimmunotherapy with anti-CD20 antibodies in B-cell non-Hodgkin's lymphomas also achieves complete responses in $75 \%$ of patients [45].

In stark contrast, PRRT has an objective response rate of around $30 \%$ (4-38\%) and is associated mostly with partial and minor remission [26, 38]; complete remission is extremely rare. It is likely that differences in tumor biology and particularly the microenvironment are of critical relevance in defining the efficacy of a radiation-targeted therapy. Clearly, the presence of a target (mainly SST receptor subtype 2) and application of a continuous (albeit decreasing) irradiation are insufficient to ensure radiation-induced cell death. The response rate to PRRT varies significantly depending on the location of the primary tumor. For example, an SST receptorpositive metastasized ileal NET exhibits a response rate which is much lower than SST receptor-positive metastasized pancreatic NET [26, 27, 29]. Given these observations, the concept of utilizing PRRT in all well to moderately differentiated gastroenteropancreatic NETs requires critical reassessment.

\section{Treatment protocol}

"A perfection of means, and confusion of aims, seems to characterize our age" (A. Einstein, The common language of science) [1].

The Rotterdam group advocates four therapeutic cycles of 7.4 GBq ${ }^{177} \mathrm{Lu}$-DOTATATE/cycle at 8 -week intervals, while the Basel group recommend two to three cycles of $100 \mathrm{mCi} /$ $\mathrm{m}^{2}{ }^{90}$ Y-DOTATOC at 4- to 6-week intervals [26, 27]. Some centers suggest a combination of both ${ }^{90}$ Y-DOTATOC and ${ }^{177}$ Lu-DOTATATE in sequence or as a cocktail to deal with small and large metastases synchronously [40]. Increase in radiosensitivity as a measure to increase the objective response rate has shown some promising results [46]. Alternatively, new agents have been proposed including the alpha particle emitting radionuclide ${ }^{213} \mathrm{Bi}$ [47]. Overall, however, there exists no general consensus on the treatment protocol, as is evident in the ambiguity of the consensus guidelines on PRRT by the European Neuroendocrine Tumor Society (ENETS) or from the International Atomic Energy Agency (IAEA) [23].

There are some major unresolved issues with PRRT protocols. These include: (a) choice of radionuclide $\left({ }^{90} \mathrm{Y}\right.$ vs ${ }^{177} \mathrm{Lu}$ vs ${ }^{213} \mathrm{Bi}$ ), (b) the cumulative and per-cycle administered activity, (c) the frequency of fractionation of radiolabeled peptides, (d) the time interval between two therapy cycles, (e) the possibility of a dosimetry-guided adaptation in administered activity, and (f) the most appropriate kidney protection regime (the options are only amino acid or in combination with 
plasma expander Gelofusine ${ }^{\circledR}$ ). In the absence of homogeneous appropriately powered prospective randomized studies, the uncertainties and unanswered questions associated with the aforementioned issues remain an obfuscating issue in the establishment of PRRT as a first-line therapy for metastasized progressive good to moderately differentiated NETs. These factors have for the most part limited PRRT administration to a handful of centers in Europe.

\section{Response evaluation}

"As the area of light expands, so does the perimeter of darkness" (attributed to A. Einstein).

Most of NET patients live with tumors for 5-10 years, making it difficult to objectively compare the efficacy of a treatment based on their survival after first diagnosis. An objective method of assessment of response based on imaging methods plays a central role in the treatment algorithm to avoid undue toxicity, improve QOL, and intervene in a timely fashion with alternative therapies in case of treatment failure.

However, an accurate measurement of response assessments, as based on two-dimensional changes in size in slow growing tumors, is a major issue. The RECIST criteria are not applicable to indolent tumors because the changes in tumor size, in response to cytotoxic or cytostatic treatment, do not become appreciable despite clinical or biochemical responses $[48,49]$. To overcome this issue, nuclear medicine physicians have advocated the use of PET-based response criteria, as a better indicator of active disease [7, 50]. Decrease in SST receptor expression on tumors as measured on ${ }^{68} \mathrm{Ga}$ DOTATOC/DOTATATE has been proposed as a superior predictor of response compared to an alteration in size. However, tumor expression of SST receptors may diminish independent of response to PRRT as for example due to transformation of G1 or G2 NET in more aggressive G3 NETs. This is one of the reasons for the lack of enthusiasm and acceptance of SST receptor expression-based response criteria by nonnuclear medicine physicians. Proponents of PRRT need to examine deeply and diligently the optimal method of response assessment to avoid the same confusion presented in the results of the Rotterdam group where the tumor-free patients (complete remission) had the same median OS as the patients with responding or stable tumor burden after PRRT [26].

\section{Conclusion}

"We can't solve problems by using the same kind of thinking we used when we created them" (A. Einstein).

The introduction of a novel therapy is always beset by difficulties: firstly, the need to persuade stakeholders in the disease space that a new option has an advantage; secondly, the need to ensure that there is clinical benefit to patients and that this is not outweighed by any downside toxicity; and thirdly, the requirement that rigorous information is provided to allow for a rational understanding of the mechanisms of action of the agent. This should be coupled to a robust assessment of efficacy. Lastly, there is the necessity to ensure that judicious interface of the new therapy with the previous modality maximizes the opportunity to provide care delivery to patients and results in accrual of advantage in disease management. PRRT has regrettably failed to meet many of these benchmarks and therefore despite its potential advantages if appropriately deployed has, for the most part, failed to completely satisfy the numerous stakeholders in the NET field. A carefully planned and judiciously managed clinical and research program by the nuclear medicine societies is needed to transform the current status of PRRT. It is clear that a diligent and judicious use of this potent therapeutic option with a proven and reliable mechanism of action, and a relatively safe toxicity profile, could potentially become the firstline therapy option for patients with metastasized progressive mild to moderately differentiated NETs.

\section{References}

1. Einstein A. Ideas and opinions. New York: Crown; 1954.

2. Modlin IM, Oberg K, Chung DC, Jensen RT, de Herder WW, Thakker RV, et al. Gastroenteropancreatic neuroendocrine tumours. Lancet Oncol 2008;9:61-72. doi:10.1016/S1470-2045(07)70410-2.

3. Weber HC. Medical treatment of neuroendocrine tumours. Curr Opin Endocrinol Diabetes Obes 2013;20:27-31. doi:10.1097/MED 0b013e32835c034f.

4. Kulke MH. Systemic therapy for advanced pancreatic neuroendocrine tumors. Semin Oncol 2013;40:75-83. doi:10.1053/j. seminoncol.2012.11.010.

5. Kulke MH, Siu LL, Tepper JE, Fisher G, Jaffe D, Haller DG, et al. Future directions in the treatment of neuroendocrine tumors: consensus report of the National Cancer Institute Neuroendocrine Tumor clinical trials planning meeting. J Clin Oncol 2011;29:934-43. doi: 10.1200/JCO.2010.33.2056.

6. Bergsma H, van Vliet EI, Teunissen JJ, Kam BL, de Herder WW, Peeters RP, et al. Peptide receptor radionuclide therapy (PRRT) for GEP-NETs. Best Pract Res Clin Gastroenterol 2012;26:867-81. doi: 10.1016/j.bpg.2013.01.004.

7. Baum RP, Kulkarni HR, Carreras C. Peptides and receptors in image-guided therapy: theranostics for neuroendocrine neoplasms. Semin Nucl Med 2012;42:190-207. doi:10.1053/j. semnuclmed.2012.01.002.

8. Modlin IM, Pavel M, Kidd M, Gustafsson BI. Review article: somatostatin analogues in the treatment of gastroenteropancreatic neuroendocrine (carcinoid) tumours. Aliment Pharmacol Ther 2010;31: 169-88. doi:10.1111/j.1365-2036.2009.04174.x.

9. Oberg K, Kvols L, Caplin M, Delle Fave G, de Herder W, Rindi G, et al. Consensus report on the use of somatostatin analogs for the management of neuroendocrine tumors of the gastroenteropancreatic system. Ann Oncol 2004;15:966-73.

10. Hofland LJ, Lamberts SW. The pathophysiological consequences of somatostatin receptor internalization and resistance. Endocr Rev 2003;24:28-47. 
11. Rinke A, Müller HH, Schade-Brittinger C, Klose KJ, Barth P, Wied $\mathrm{M}$, et al. Placebo-controlled, double-blind, prospective, randomized study on the effect of octreotide LAR in the control of tumor growth in patients with metastatic neuroendocrine midgut tumors: a report from the PROMID Study Group. J Clin Oncol 2009;27:4656-63.

12. Kidd M, Schally AV, Pfragner R, Malfertheiner MV, Modlin IM. Inhibition of proliferation of small intestinal and bronchopulmonary neuroendocrine cell lines by using peptide analogs targeting receptors. Cancer 2008;112:1404-14.

13. Kidd M, Drozdov I, Joseph R, Pfragner R, Culler M, Modlin I. Differential cytotoxicity of novel somatostatin and dopamine chimeric compounds on bronchopulmonary and small intestinal neuroendocrine tumor cell lines. Cancer 2008;113:690-700. doi:10.1002/ cncr.23700.

14. Susini C, Buscail L. Rationale for the use of somatostatin analogs as antitumor agents. Ann Oncol 2006;17:1733-42.

15. Li SC, Martijn C, Cui T, Essaghir A, Luque RM, Demoulin JB, et al. The somatostatin analogue octreotide inhibits growth of small intestine neuroendocrine tumour cells. PLoS One 2012;7:e48411. doi:10. 1371/journal.pone.0048411.

16. Cescato R, Loesch KA, Waser B, Mäcke HR, Rivier JE, Reubi JC, et al. Agonist-biased signaling at the sst2A receptor: the multisomatostatin analogs KE108 and SOM230 activate and antagonize distinct signaling pathways. Mol Endocrinol 2010;24:240-9. doi:10. 1210/me.2009-0321.

17. Caplin M, Ruszniewski P, Pavel M, et al. A randomized double-blind placebo-controlled study of lanreotide antiproliferative response in patients with enteropancreatic neuroendocrine tumours (CLARINET). 17th ECCO-38th ESMO-32nd ESTRO European Cancer Congress. Amsterdam; 2013. p. Abstract E17-7103.

18. Wolin EM. PI3K/Akt/mTOR pathway inhibitors in the therapy of pancreatic neuroendocrine tumors. Cancer Lett 2013;335:1-8.

19. Faivre S, Castellano D, Strosberg J, Gonzalez E, Salazar R. Pancreatic NETs: where do we stand now? Cancer Metastasis Rev 2014;33:361-6.

20. Dahan L, Bonnetain F, Rougier P, Raoul JL, Gamelin E, Etienne PL, et al. Phase III trial of chemotherapy using 5-fluorouracil and streptozotocin compared with interferon alpha for advanced carcinoid tumors: FNCLCC-FFCD 9710. Endocr Relat Cancer 2009;16:135161.

21. Strosberg JR, Fine RL, Choi J, Nasir A, Coppola D, Chen DT, et al. First-line chemotherapy with capecitabine and temozolomide in patients with metastatic pancreatic endocrine carcinomas. Cancer 2011;117:268-75. doi:10.1002/cncr.25425.

22. Strosberg JR, Fisher GA, Benson AB, Malin JL, GEPNET Treatment Consensus Panel, Cherepanov D, Broder MS, et al. Systemic treatment in unresectable metastatic well-differentiated carcinoid tumors: consensus results from a modified delphi process. Pancreas 2013;42: 397-404. doi:10.1097/MPA.0b013e31826d3a17.

23. Bodei L, Mueller-Brand J, Baum RP, Pavel ME, Horsch D, O'Dorisio MS, et al. Erratum to: the joint IAEA, EANM, and SNMMI practical guidance on peptide receptor radionuclide therapy (PRRNT) in neuroendocrine tumours. Eur J Nucl Med Mol Imaging 2014;41:584. doi:10.1007/s00259-013-2454-3.

24. Tubiana M, Dutreix J, Wambersie A, editors. Introduction to radiobiology. Intrinsic cellular radiosensitivity, chap. 4.4. London: Taylor \& Francis; 1990.

25. Pauwels S, Barone R, Walrand S, Borson-Chazot F, Valkema R, Kvols LK, et al. Practical dosimetry of peptide receptor radionuclide therapy with (90)Y-labeled somatostatin analogs. J Nucl Med 2005;46:92S-8S.

26. Kwekkeboom DJ, de Herder WW, Kam BL, van Eijck CH, van Essen M, Kooij PP, et al. Treatment with the radiolabeled somatostatin analog [177 Lu-DOTA 0, Tyr3]octreotate: toxicity, efficacy, and survival. J Clin Oncol 2008;26:2124-30.
27. Imhof A, Brunner $P$, Marincek N, Briel M, Schindler C, Rasch $\mathrm{H}$, et al. Response, survival, and long-term toxicity after therapy with the radiolabeled somatostatin analogue [90Y-DOTA]-TOC in metastasized neuroendocrine cancers. J Clin Oncol 2011;29:2416-23.

28. Ruszniewski P, Ish-Shalom S, Wymenga M, O’Toole D, Arnold R, Tomassetti $\mathrm{P}$, et al. Rapid and sustained relief from the symptoms of carcinoid syndrome: results from an open 6-month study of the 28-day prolonged-release formulation of lanreotide. Neuroendocrinology 2004;80:244-51.

29. Bushnell Jr DL, O’Dorisio TM, O’Dorisio MS, Menda Y, Hicks RJ, Van Cutsem E, et al. 90Y-edotreotide for metastatic carcinoid refractory to octreotide. J Clin Oncol 2010;28:1652-9.

30. Khan S, Krenning EP, van Essen M, Kam BL, Teunissen JJ, Kwekkeboom DJ. Quality of life in 265 patients with gastroenteropancreatic or bronchial neuroendocrine tumors treated with [177Lu-DOTA0,Tyr3]octreotate. J Nucl Med 2011;52:1361-8.

31. Bodei L, Ferone D, Grana CM, Cremonesi M, Signore A, Dierckx RA, et al. Peptide receptor therapies in neuroendocrine tumors. J Endocrinol Invest 2009;32:360-9.

32. Valkema R, Pauwels S, Kvols LK, Barone R, Jamar F, Bakker WH, et al. Survival and response after peptide receptor radionuclide therapy with [90Y-DOTA0, Tyr3]octreotide in patients with advanced gastroenteropancreatic neuroendocrine tumors. Semin Nucl Med 2006;36:147-56.

33. Kuzel T, Rosen S. Radioimmunotherapy of lymphomas and leukemias. In: Henkin R, Boles M, Dillehay G, editors. Nuclear medicine. St. Louis: Mosby; 1996. p. 594-600.

34. Valkema R, Pauwels SA, Kvols LK, Kwekkeboom DJ, Jamar F, de Jong $\mathrm{M}$, et al. Long-term follow-up of renal function after peptide receptor radiation therapy with $(90)$ Y-DOTA $(0), \operatorname{Tyr}(3)$-octreotide and (177)Lu-DOTA(0), Tyr(3)-octreotate. J Nucl Med 2005;46: 83S-91S.

35. Bodei L, Cremonesi M, Ferrari M, Pacifici M, Grana CM, Bartolomei $\mathrm{M}$, et al. Long-term evaluation of renal toxicity after peptide receptor radionuclide therapy with 90Y-DOTATOC and 177Lu-DOTATATE: the role of associated risk factors. Eur J Nucl Med Mol Imaging 2008;35:1847-56.

36. Sandström M, Garske-Román U, Granberg D, Johansson S, Widström C, Eriksson B, et al. Individualized dosimetry of kidney and bone marrow in patients undergoing 177Lu-DOTA-octreotate treatment. J Nucl Med 2013;54:33-41. doi:10.2967/jnumed.112. 107524.

37. Kwekkeboom DJ, Mueller-Brand J, Paganelli G, Anthony LB, Pauwels S, Kvols LK, et al. Overview of results of peptide receptor radionuclide therapy with 3 radiolabeled somatostatin analogs. J Nucl Med 2005;46:62S-6S.

38. Bodei L, Cremonesi M, Grana CM, Fazio N, Iodice S, Baio SM, et al. Peptide receptor radionuclide therapy with (177)Lu-DOTATATE: the IEO phase I-II study. Eur J Nucl Med Mol Imaging 2011;38:212535.

39. Kwekkeboom D. EANM. In: Bodei L, editor.; 2013.

40. Pfeifer AK, Gregersen T, Grønbæk H, Hansen CP, MüllerBrand J, Herskind Bruun K, et al. Peptide receptor radionuclide therapy with Y-DOTATOC and (177)Lu-DOTATOC in advanced neuroendocrine tumors: results from a Danish cohort treated in Switzerland. Neuroendocrinology 2011;93:189-96. doi:10.1159/000324096.

41. Sabet A, Ezziddin K, Pape UF, Ahmadzadehfar H, Mayer K, Pöppel $\mathrm{T}$, et al. Long-term hematotoxicity after peptide receptor radionuclide therapy with 177Lu-octreotate. J Nucl Med 2013;54:1857-61. doi: 10.2967/jnumed.112.119347.

42. Bodei L, Kidd M, Paganelli G, Grana C, Drozdov I, Cremonesi M, et al. Clinical features are not reliable in predicting long-term toxicity after PRRT - evidence from $>800$ patients to support genetic screen development. Barcelona: ENETs; 2014. 
43. Seaborg GT. My career as a radioisotope hunter. JAMA 1995;273: 961-4.

44. Luster M, Hänscheid H, Freudenberg LS, Verburg FA. Radioiodine therapy of metastatic lesions of differentiated thyroid cancer. J Endocrinol Invest 2012;35:21-9.

45. Goldsmith SJ. Radioimmunotherapy of lymphoma: Bexxar and Zevalin. Semin Nucl Med 2010;40:122-35. doi:10.1053/j. semnuclmed.2009.11.002.

46. Claringbold PG, Brayshaw PA, Price RA, Turner JH. Phase II study of radiopeptide $177 \mathrm{Lu}$-octreotate and capecitabine therapy of progressive disseminated neuroendocrine tumours. Eur J Nucl Med Mol Imaging 2011;38:302-11. doi:10.1007/ s00259-010-1631-x.

47. Giesel FL, Wulfert S, Zechmann CM, Haberkorn U, Kratochwil C, Flechsig P, et al. Contrast-enhanced ultrasound monitoring of perfusion changes in hepatic neuroendocrine metastases after systemic versus selective arterial $177 \mathrm{Lu} /$ 90Y-DOTATOC and 213Bi-DOTATOC radiopeptide therapy. Exp Oncol 2013;35:122-6.

48. de Mestier L, Dromain C, d'Assignies G, Scoazec JY, Lassau N, Lebtahi R, et al. Evaluating neuroendocrine tumors progression and therapeutic response: state of the art. Endocr Relat Cancer 2013. doi: 10.1530/ERC-13-0365.

49. van Vliet EI, Krenning EP, Teunissen JJ, Bergsma H, Kam BL, Kwekkeboom DJ. Comparison of response evaluation in patients with gastroenteropancreatic and thoracic neuroendocrine tumors after treatment with [177Lu-DOTA0, Tyr3]octreotate. J Nucl Med 2013;54:1689-96. doi:10.2967/jnumed.112.117408.

50. Wahl RL, Jacene H, Kasamon Y, Lodge MA. From RECIST to PERCIST: evolving considerations for PET response criteria in solid tumors. J Nucl Med 2009;50:122S-50S. doi:10.2967/jnumed.108. 057307. 